\title{
Exploratory Approach to Performance of Smart Components of Intelligent [Smart] Buildings
}

International Conference on Human Systems Engineering and Design: Future Trends and Applications

IHSED 2020: Human Systems Engineering and Design III pp 215-224 | Cite as

- Lekan Amusan (1) Email author (lekan.amusan@covenantuniversity.edu.ng)

- Emetere Moses (2)

- Ojelabi Rapheal (3)

1. Lekan Amusan Building Technology Department College of Science and Technology, Covenant University, , Ota, Nigeria

2. Moses Emetere Department of Physics, Covenant University, , Ota, Nigeria

3. Ojelabi Rapheal Building Technology Department College of Science and Technology, Covenant University, , Ota, Nigeria

Conference paper

First Online: 30 August 2020

- 369 Downloads

Part of the Advances in Intelligent Systems and Computing book series (AISC, volume 1269)

\section{Abstract}

Intelligent building is gaining access to the construction industry all over the world in recent times. Building functionality and the need for occupant comfort has been the major drive toward advent of intelligent Buildings. In the light of this, there is a need to carry out continuous evaluation of performance of the building to ensure value for money. In this study therefore, post-occupation building managers at different levels of types of buildings were censored within the scope of the following parameters; awareness on current state of practice in office intelligent building application, the level of satisfaction of managers and the users of intelligent buildings' performance, effectiveness of the security system and access control in building and performance enhancement measures to be adopted and factors that influences effective performance of intelligent building accessories. Survey method was used with the aid of questionnaire the resultant data was analyse using relative agreement index (RAI), Mann-Whitney U-Test, Pearsons's Chi-square test and Student's T-test. The study presented factors that influences integration of automated accessory in building and their performance, effectiveness of security system and access control, conventional automation application 
in building products among others. The study recommend constructive innovative approach to intelligent building management, strategic plan should be instituted in maintenance operation, proactive management building accessory, and collaborative stake holder inclusive management, as panacea to forestalling negative attendant consequence of facility negligence in intelligent building.

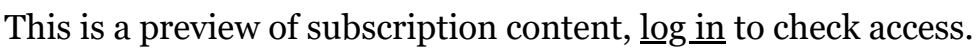

\section{References}

1. Kubba, S.: Green project commissioning. In Handbook of Green Building Design and Construction LEED, BREEAM, and Green Globes, Butterworth -Heinemann, Oxford (2017)

Google Scholar (https://scholar.google.com/scholar?

q=Kubba\%2C\%2OS.\%3A\%2OGreen\%2Oproject\%2Ocommissioning.\%2OIn\%2OHa ndbook\%20of\%20Green\%2oBuilding\%2oDesign\%20and\%20Construction\%20LE ED\%2C\%2OBREEAM\%2C\%2Oand\%20Green\%20Globes\%2C\%2oButterworth\%2 0\%E2\%80\%93Heinemann\%2C\%20Oxford\%20\%282017\%29)

2. Altomonte, S., Saadouni, S., Kent, M.G., Schiavon, S.: Satisfaction with Indoor environmental quality in BREEAM and Non-BREEAM certified office buildings. Archit. Sci. Rev. 6o, 343-355 (2017). https://doi.org/10.1080/00038628.2017.1336983 (https://doi.org/10.1080/00038628.2017.1336983)

3. Bhavani, R.G., Mohen, M.: Awareness on lighting energy saving and user satisfaction in residential and office buildings. In: International Conference on Biological, Civil and Environmental Engineering (BCEE-2014), Dubai (UAE), 1718 March 2014 (2014)

Google Scholar (https://scholar.google.com/scholar? q=Bhavani\%2C\%2oR.G.\%2C\%20Mohen\%2C\%20M.\%3A\%20Awareness\%20on\% 2olighting\%20energy\%20saving\%20and\%20user\%20satisfaction\%20in\%20resid ential\%20and\%20office\%2obuildings.\%20In\%3A\%2oInternational\%20Conferenc e\%20on\%2oBiological\%2C\%2oCivil\%2Oand\%20Environmental\%2oEngineering $\% 20 \% 28 \mathrm{BCEE}-$ 2014\%29\%2C\%20Dubai\%20\%28UAE\%29\%2C\%2017\%E2\%80\%9318\%20March \%202014\%20\%282014\%29)

4. Hoseini, G., Dahlan, N.D, Berardi, U., Hoseini, G.A., Makareni, N.: Sustainable energy performance of green buildings: a review of current theory. Renew.

Sustain. Energy Rev. 25(1), 1-7 (2013)

Google Scholar (https://scholar.google.com/scholar? q=Hoseini\%2C\%20G.\%2C\%2oDahlan\%2C\%20N.D\%2C\%2oBerardi\%2C\%2oU.\% 2C\%20Hoseini\%2C\%20G.A.\%2C\%2oMakareni\%2C\%20N.\%3A\%2oSustainable\% 20energy\%2operformance\%20of\%2ogreen\%2obuildings\%3A\%20a\%2oreview\%2 oof\%20current\%20theory.\%20Renew.\%2oSustain.\%2oEnergy\%2oRev.\%2025\%2 81\%29\%2C\%201\%E2\%80\%937\%20\%282013\%29)

5. Sherif, K.: The Use of Intelligent Buildings to Achieve Sustainability through Architectural Proposal (2011)

Google Scholar (https://scholar.google.com/scholar? q=Sherif\%2C\%20K.\%3A\%20The\%20Use\%20of\%2oIntelligent\%2oBuildings\%2ot 
0\%20Achieve\%20Sustainability\%2othrough\%20Architectural\%2oProposal\%20\% 282011\%29)

6. Brooks, D.J.: Intelligent buildings: an investigation into current and emerging security vulnerabilities in automated building systems using an applied defeat methodology. (2012). https://doi.org/10.4018/978-1-4666-2659-1.choo1

(https://doi.org/10.4018/978-1-4666-2659-1.cho01)

CrossRef (https://doi.org/10.4018/978-1-4666-2659-1.choo1)

Google Scholar (http://scholar.google.com/scholar_lookup?

\&author=DJ.\%20Brooks\&journal=Intelligent\%2obuildings\%3A\%20an\%2oinvesti gation\%20into\%20current\%20and\%20emerging\%20security\%2ovulnerabilities\% 20in\%20automated\%2obuilding\%20systems\%20using\%20an\%20applied\%2odef eat\%20methodology.\&publication_year=2012\&doi=10.4018\%2F978-1-46662659-1.choo1)

7. Ai-Abbas, S.H.: Energy conservation in residential building using green architecture principles. Master Thesis. World SB 14 Conference, Cairo University, Egypt (2011)

Google Scholar (https://scholar.google.com/scholar?q=Ai-

Abbas\%2C\%20S.H.\%3A\%2OEnergy\%2Oconservation\%2oin\%2oresidential\%2obu ilding\%20using\%2ogreen\%20architecture\%2oprinciples.\%2oMaster\%20Thesis.\% 20World\%20SB\%2014\%20Conference\%2C\%20Cairo\%20University\%2C\%20Egyp t\%20\%282011\%29)

8. Lekan, A., Dolapo, D., Joshua, O.: Cost and time performance of construction projects. Int. J. Mech. Eng. Technol. (IJMET) 8(10), 918-927 (2017)

Google Scholar (http://scholar.google.com/scholar_lookup?

title=Cost\%20and\%20time\%2operformance\%20of\%20construction\%2oprojects\& author=A.\%20Lekan\&author=D.\%20Dolapo\&author=O.\%2OJoshua\&journal=Int. \%20J.\%20Mech.\%20Eng.\%20Technol.\%20\%28IJMET\%29\&volume=8\&issue=10 \&pages $=918-927 \&$ publication_year $=2017)$

9. Manic, M., Rodriguez-Andina, J.J., Amarasinghe, K., Reiger, C.G.: Intelligent buildings of the future: cyberaware, deep learning powered, and human interacting. IEEE Ind. Electron. Mag. (2016) Google Scholar (https://scholar.google.com/scholar? q=Manic\%2C\%20M.\%2C\%2oRodriguezAndina\%2C\%2OJ.J.\%2C\%2OAmarasinghe\%2C\%20K.\%2C\%2oReiger\%2C\%2OC.G .\%3A\%2oIntelligent\%2obuildings\%20of\%2othe\%2ofuture\%3A\%20cyberaware\% 2C\%20deep\%2olearning\%2opowered\%2C\%20and\%2ohuman\%2ointeracting.\%2 oIEEE\%2OInd.\%20Electron.\%20Mag.\%20\%282016\%29)

10. Brooks, D.J., Coole, M., Haskell-Dowland, P., Griffiths, M., Lockhart, N.: Building Automation \& Control Systems: An Investigation into Vulnerabilities, Current Practice \& Security Management Best Practice. ASIS Foundation Project (2017) Google Scholar (https://scholar.google.com/scholar? q=Brooks\%2C\%20D.J.\%2C\%20Coole\%2C\%20M.\%2C\%2oHaskellDowland\%2C\%2OP.\%2C\%20Griffiths\%2C\%20M.\%2C\%2OLockhart\%2C\%2ON.\%3 A\%20Building\%20Automation\%20\%26\%20Control\%20Systems\%3A\%20An\%20I nvestigation\%20into\%2oVulnerabilities\%2C\%20Current\%20Practice\%20\%26\%2 oSecurity\%20Management\%2oBest\%20Practice.\%20ASIS\%20Foundation\%20Pr oject\%20\%282017\%29) 
11. Osawaru, F., Amusan, L., Awotinde, O., Akanya, C., Asiyanbola, O., Akinbo, F.: Planning materials supply for construction works. In: 22nd International Conference on Science and Sustainable Development, ICSSD 2018: The Role of Science in Novel Research and Advances in Technology; Center for Research, Innovation and Discovery, Covenant University (CUCRID), Nigeria, 14 May 2018 through 16 May 2018, Code 138212 (2018)

Google Scholar (https://scholar.google.com/scholar? q=Osawaru\%2C\%20F.\%2C\%20Amusan\%2C\%20L.\%2C\%2OAwotinde\%2C\%2oO. \%2C\%20Akanya\%2C\%20C.\%2C\%2OAsiyanbola\%2C\%20O.\%2C\%2OAkinbo\%2C\% 2OF.\%3A\%2OPlanning\%2omaterials\%20supply\%2ofor\%2oconstruction\%2Owork s.\%20In\%3A\%2022nd\%2oInternational\%2oConference\%20on\%20Science\%20an d\%20Sustainable\%2oDevelopment\%2C\%20ICSSD\%202018\%3A\%20The\%20Rol e\%20of\%20Science\%20in\%20Novel\%2oResearch\%20and\%2oAdvances\%20in\%2 oTechnology\%3B\%2oCenter\%2ofor\%2oResearch\%2C\%2oInnovation\%20and\%2 oDiscovery\%2C\%20Covenant\%2oUniversity\%20\%28CUCRID\%29\%2C\%20Nigeri a\%2C\%2014\%20May\%202018\%20through\%2016\%20May\%202018\%2C\%2oCod e\%20138212\%20\%282018\%29)

12. Lekan, A., Clinton, A., Blossom,O., Babatunde, O.: Building informatics: post occupancy narratives of automation component of intelligent and non intelligent buildings. Int. J. Pharm. Res. 6(5) (2020)

Google Scholar (https://scholar.google.com/scholar? q=Lekan\%2C\%2OA.\%2C\%2OClinton\%2C\%2oA.\%2C\%2oBlossom\%2CO.\%2C\%20 Babatunde\%2C\%20O.\%3A\%20Building\%2oinformatics\%3A\%20post\%20occupan cy\%20narratives\%20of\%20automation\%20component\%20of\%2ointelligent\%20a nd\%20non\%2ointelligent\%2obuildings.\%2oInt.\%20J.\%20Pharm.\%20Res.\%206 $\% 285 \% 29 \% 20 \% 282020 \% 29)$

13. Clements-Croome, T.D.J.: What do we mean by intelligent buildings. Autom. Constr. 6(5/6), 395-400 (1997)

Google Scholar (http://scholar.google.com/scholar_lookup?

title=What\%20do\%20we\%2omean\%2oby\%2ointelligent\%2obuildings\&author=T DJ.\%20Clements-

Croome\&journal=Autom.\%20Constr.\&volume $=6 \&$ issue $=5 \% 2 \mathrm{~F} 6 \&$ pages $=395-$ 400\&publication_year=1997)

14. Yiu, C.Y., Ho, C.W., Lo, S.M.: Weathering effects on external wall tiling systems. Constr. Build. Mater. 21(3), 594-600 (2007)

CrossRef (https://doi.org/10.1016/j.conbuildmat.2005.11.002)

Google Scholar (http://scholar.google.com/scholar_lookup?

title=Weathering\%20effects\%20on\%20external\%20wall\%2otiling\%20systems\&a uthor=CY.\%2OYiu\&author $=$ CW.\%2OHo\&author=SM.\%2OLo\&journal=Constr.\%2 oBuild.\%20Mater. $\&$ volume $=21$ \&issue $=3 \&$ pages $=594-$

6oo\&publication_year=2007)

15. Harris, S.Y.: Building Pathology: Deterioration, Diagnostics, and Intervention. John Wiley \& Sons Inc., New York (2001) Google Scholar (http://scholar.google.com/scholar_lookup? title=Building\%20Pathology\%3A\%20Deterioration\%2C\%2oDiagnostics\%2C\%20a nd\%20Intervention\&author=SY.\%20Harris\&publication_year=2001)

16. BSI (1991 BS4778-3.1: 1991) Quality Vocabulary: Part 3: Availability, Reliability and Maintainability Terms, Section 3.1 Guide to Concepts and Related Definitions, 
British Standards Institution, UK (1991)

Google Scholar (https://scholar.google.com/scholar?

q=BSI\%20\%281991\%20BS4778-

3.1\%3A\%201991\%29\%20Quality\%20Vocabulary\%3A\%2oPart\%203\%3A\%20Avail ability\%2C\%2oReliability\%20and\%2oMaintainability\%20Terms\%2C\%2oSection

\%C2\%A03.1\%2oGuide\%20to\%20Concepts\%20and\%2oRelated\%2oDefinitions\%2 C\%20British\%20Standards\%20Institution\%2C\%20UK\%20\%281991\%29)

17. Seeley, I.H.: Building Maintenance, 2nd edn. Macmillan, Basingstoke (1992)

Google Scholar (http://scholar.google.com/scholar_lookup?

title=Building\%2OMaintenance\&author=IH.\%20Seeley\&publication_year=1992)

18. Mozer, M.C.: An intelligent environment must be adaptive. IEEE Intell. Syst.

Appl. 14(2), 11-13 (1999)

CrossRef (https://doi.org/10.1109/MIS.1999.757623)

Google Scholar (http://scholar.google.com/scholar_lookup?

title=An\%20intelligent\%2oenvironment\%20must\%2obe\%20adaptive\&author=M

C.\%20Mozer\&journal=IEEE\%2oIntell.\%20Syst.\%20Appl.\&volume=14\&issue=2\&

pages $=11-13 \&$ publication_year $=1999)$

19. Harrison, A., Loe, E., Read, J. (eds.): Intelligent Buildings in South East Asia. E \& FN Spon, London (1998)

Google Scholar (https://scholar.google.com/scholar?

q=Harrison\%2C\%20A.\%2C\%2oLoe\%2C\%2oE.\%2C\%2oRead\%2C\%2OJ.\%20\%28e

ds.\%29\%3A\%2OIntelligent\%2oBuildings\%20in\%20South\%2oEast\%20Asia.\%20E

\%20\%26\%20FN\%20Spon\%2C\%20London\%20\%281998\%29)

20. Raouf, A.M., Al-Ghamdi, S.G.: Framework to optimize cost of quality in delivering and operating green buildings. In: Proceedings of International Conference on Sustainable Infrastructure 2019, Los Angeles, CA, USA, 6-9 November 2019, pp. 338-347 (2019). https://doi.org/10.1061/9780784482650.035

(https://doi.org/10.1061/9780784482650.035)

\section{Copyright information}

(C) The Editor(s) (if applicable) and The Author(s), under exclusive license to Springer Nature Switzerland AG 2021

\section{About this paper}

Cite this paper as:

Amusan L., Moses E., Rapheal O. (2021) Exploratory Approach to Performance of Smart Components of Intelligent [Smart] Buildings. In: Karwowski W., Ahram T., Etinger D., Tanković N., Taiar R. (eds) Human Systems Engineering and Design III. IHSED 2020. Advances in Intelligent Systems and Computing, vol 1269. Springer, Cham. https://doi.org/10.1007/978-3-030-58282-1_35

- First Online 30 August 2020

- DOI https://doi.org/10.1007/978-3-030-58282-1_35

- Publisher Name Springer, Cham

- Print ISBN 978-3-030-58281-4 
- Online ISBN 978-3-030-58282-1

- eBook Packages Engineering Engineering_(Ro).

- Buy this book on publisher's site

- Reprints and Permissions

\section{Personalised recommendations}

\section{SPRINGER NATURE}

(C) 2020 Springer Nature Switzerland AG. Part of $\underline{\text { ppringer Nature. }}$

Not logged in Not affiliated 165.73.192.252 